\title{
Unambiguous discrimination between two rank-2 mixed quantum states
}

\author{
Mihailo Stojnic \\ Department of Electrical Engineering \\ California Institute of Technology \\ Pasadena CA 91125, USA \\ Email:mihailo@systems.caltech.edu
}

\author{
Babak Hassibi \\ Department of Electrical Engineering \\ California Institute of Technology \\ Pasadena CA 91125, USA \\ Email: hassibi@systems.caltech.edu
}

\begin{abstract}
In this paper we consider the problem of the optimal quantum unambiguous detection between two mixed quantum states. More specifically, we consider two mixed quantum states of rank 2 which lie in a Hilbert space of dimension 4. Using duality theory we explicitly characterize the optimal measurement operators. Furthermore, as a by-product of our framework we obtain a closed form solution of unambiguous discrimination between a pure and a mixed quantum state.
\end{abstract}

\section{INTRODUCTION}

Quantum unambiguous detection is a somewhat recent approach in distinguishing among a collection of quantum states. It was initially introduced in [1] and further considered in [2],[3]. The main idea was to allow for an inconclusive result of the measurement procedure. In return, if the measurement produces an answer then that answer is correct with probability 1. An interesting variation of this approach is maximization of the probability of correct detection ([9],[10]). This approach attracted significant attention in the last several years (for a recent survey on the topic see e.g. [5]).

Bounds on the efficiency (the maximum probability of correct detection) of unambiguous discrimination and conditions for achieving them in different scenarios were studied in [15],[7],[16],[14],[12]. Applications of semi-definite programming in finding the optimal measurement operators were considered in [8],[18],[17]. In this paper, we restrict ourselves to a specific case of unambiguous discrimination of two mixed quantum states. A special case of this problem, when one of the states is pure and the other one is mixed, was solved analytically in [4]. Additionally, in [7] a bound on maximal probability of correct detection in the case of unambiguous discrimination of two general mixed states was derived. Furthermore it was shown that the bound is tight in the case when one of the states is pure, thus matching the result of [4] obtained in the context of quantum filtering. In [6] the authors derive an analytical solution for unambiguous discrimination of a special class of two mixed states. Namely, the authors analyze the case when two mixed states are uniformly mixed, i.e. when their representations in Jordan bases correspond to their spectral representations. In [13] another special case of two quantum states connected by a unitary transformation is linked to the previous one and solved analytically as well. However, in the most general case, analytically solving the unambiguous discrimination of two mixed states still remains a very difficult task. It is interesting to note that in [11], the authors showed that the problem of unambiguous discrimination of any two mixed states can always be reduced to the problem of distinguishing two states of rank $d$ that lie in a $2 d$-dimensional Hilbert space. It should also be noted that in [6], the authors emphasized the incredible difficulty of solving that problem for an arbitrary $d$, while still believing that the case when $d=2$ may be within the reach. In this paper we solve the problem when $d=2$.

\section{PRoblem Formulation}

Assume that we have two quantum states $\rho_{1}$ and $\rho_{2}$. Further, assume that their rank is 2 and that they lie in a 4-dimensinal Hilbert space. The quantum unambiguous detection technique assumes the existence of three measurement operators $\left\{\Pi_{i}, 0 \leq i \leq 2\right\}$ that satisfy

$$
\Pi_{i}=\Pi_{i}^{*} \geq 0, \quad 0 \leq i \leq 2 ; \quad \sum_{i=0}^{2} \Pi_{i}=I .
$$

As usual $\operatorname{Tr}\left(\rho_{j} \Pi_{i}\right), i>0$ represents the probability that if the system is prepared in state $\rho_{j}$ the detected state is $\rho_{i}$. Since unambiguous discrimination doesn't allow for incorrect detection it must hold

$$
\operatorname{Tr}\left(\rho_{j} \Pi_{i}\right)=0, \quad 1 \leq i, j \leq 2, i \neq j .
$$

From (2) it is clear that the eigenvectors of $\Pi_{1}$ have to be in the null space of $\rho_{2}$ and the eigenvectors of $\Pi_{2}$ have to be in the null space of $\rho_{1}$. Let $\Theta_{2}$ be the matrix whose column represents an orthonormal basis of the null space of $\rho_{1}$, and analogously $\Theta_{1}$ be the matrix whose columns represent an orthonormal basis of the null space of $\rho_{2}$. Clearly, $\Theta_{1}$, $\Theta_{2}$ are $4 \times 2$ matrices. Using the introduced matrices $\Theta$ 's we can represent the measurement operators of interest as $\Pi_{i}=\Theta_{i} \Delta_{i} \Theta_{i}^{*}, i=1,2$, where $\Delta_{i}=\Delta_{i}^{*} \geq 0$ and the $\Delta$ 's are of the corresponding dimensions. It is then easy to see that maximizing the probability of correct detection is equivalent to solving (see e.g. [13], [6],[7],[18])

$$
\begin{aligned}
\max _{\Delta_{1}=\Delta_{1}^{*} \geq 0, \Delta_{2}=\Delta_{2}^{*} \geq 0} & \sum_{i=1}^{2} p_{i} \operatorname{Tr}\left(\rho_{i} \Theta_{i} \Delta_{i} \Theta_{i}^{*}\right) \\
\text { subject to } & \Theta_{1} \Delta_{1} \Theta_{1}^{*}+\Theta_{2} \Delta_{2} \Theta_{2}^{*} \leq I .
\end{aligned}
$$

where $p_{i}, 1 \leq i \leq 2$ is the a priori probability that system was prepared in state $i$. As shown in [18] the dual of the previous 
primal problem can be written as

$$
\begin{aligned}
\min _{Z=Z^{*}} & \operatorname{Tr}(Z) \\
\text { subject to } & \Theta_{i}^{*}\left(Z-p_{i} \rho_{i}\right) \Theta_{i} \geq 0, \quad 1 \leq i \leq 2 \\
& Z \geq 0 .
\end{aligned}
$$

Denoting by $D_{i}=\Theta_{i}^{*} p_{i} \rho_{i} \Theta_{i}, i=1,2$ we get the following formulation of the dual problem

$$
\begin{aligned}
\min _{Z=Z^{*}} & \operatorname{Tr}(Z) \\
\text { subject to } & \Theta_{i}^{*} Z \Theta_{i} \geq D_{i}, \quad 1 \leq i \leq 2 ; \\
& Z \geq 0 .
\end{aligned}
$$

In order to solve (3) we will first solve the dual problem and then find the solution of the primal based on the conclusions about the optimality conditions given in [18].

\section{THE DUAL PROBLEM}

It is easy to see that the problem in (4) is equivalent to

$$
\begin{aligned}
\min _{Z=Z^{*}, m_{1}, m_{2}} & \operatorname{Tr}(Z) \\
\text { subject to } & \Theta_{1}^{*} Z \Theta_{1}=D_{1}+m_{1} m_{1}^{*} \\
& \Theta_{2}^{*} Z \Theta_{2}=D_{2}+m_{2} m_{2}^{*} \\
& Z \geq 0 .
\end{aligned}
$$

where $m_{1}, m_{2}$ are $2 \times 2$ matrices. Denote by $\Theta_{2}^{\perp}$ a $4 \times 2$ matrix such that $\left[\begin{array}{ll}\Theta_{2} & \Theta_{2}^{\perp}\end{array}\right]\left[\begin{array}{ll}\Theta_{2} & \Theta_{2}^{\perp}\end{array}\right]^{*}=I$ and by $F$ and $E 2 \times 2$ matrices such that $\Theta_{1}=\Theta_{2} F+\Theta_{2}^{\perp} E$. Since, $Z$ is Hermitian we can write $Z=A A^{*}$ where $A$ is some $4 \times 4$ matrix (it can in fact be shown that in case of optimal $Z, A$ can even be represented as $4 \times 2$ matrix). Then the second constraint in (5) becomes

$$
\Theta_{2}^{*} A A^{*} \Theta_{2}=D_{2}+m_{2} m_{2}^{*}
$$

From (6) we get

$$
A=\Theta_{2} \sqrt{D_{2}+m_{2} m_{2}^{*}} K+\Theta_{2}^{\perp} S
$$

where $K$ is a $2 \times 4$ matrix such that $K K^{*}=I, \sqrt{D_{2}+m_{2} m_{2}^{*}}$ is any positive square root of the Hermitian matrix $D_{2}+$ $m_{2} m_{2}^{*}$, and $S$ is any $2 \times 4$ matrix. From the first constraint in (5) we have

$$
\Theta_{1}^{*} A A^{*} \Theta_{1}=D_{1}+m_{1} m_{1}^{*}
$$

and

$$
\Theta_{1}^{*} A=\sqrt{D_{1}+m_{1} m_{1}^{*}} L
$$

where $L$ is a $2 \times 4$ matrix such that $L L^{*}=I$, and $\sqrt{D_{1}+m_{1} m_{1}^{*}}$ is any positive square root of the Hermitian matrix $D_{1}+m_{1} m_{1}^{*}$. Using the representation of $\Theta_{1}$ given earlier and $A$ obtained in (7) we have

$$
\begin{aligned}
\Theta_{1}^{*} A & =\Theta_{1}^{*}\left(\Theta_{2} \sqrt{D_{2}+m_{2} m_{2}^{*}} K+\Theta_{2}^{\perp} S\right) \\
& =F^{*} \sqrt{D_{2}+m_{2} m_{2}^{*}} K+E^{*} S
\end{aligned}
$$

Now, replacing result from (9) in (8) we get

$$
F^{*} \sqrt{D_{2}+m_{2} m_{2}^{*}} K+E^{*} S=\sqrt{D_{1}+m_{1} m_{1}^{*}} L
$$

From now on, in order to avoid tedious discussion of degenerative low rank cases we will assume that $E$ is invertible. Then from (10) we easily have

$$
S=E^{-*} \sqrt{D_{1}+m_{1} m_{1}^{*}} L-E^{-*} F^{*} \sqrt{D_{2}+m_{2} m_{2}^{*}} K
$$

Using the expression for $S$ form (11) we have

$$
\begin{aligned}
\operatorname{Tr} Z & =\operatorname{Tr} S S^{*}+\operatorname{Tr}\left(D_{2}+m_{2} m_{2}^{*}\right) \\
& =\operatorname{Tr}\left(E^{-*}\left(D_{1}+m_{1} m_{1}^{*}\right) E^{-1}\right) \\
& -\operatorname{Tr}\left(E^{-*} \sqrt{D_{1}+m_{1} m_{1}^{*}} L K^{*} \sqrt{D_{2}+m_{2} m_{2}^{*}} F E^{-1}\right) \\
& -\operatorname{Tr}\left(E^{-*} F^{*} \sqrt{D_{2}+m_{2} m_{2}^{*}} K L^{*} \sqrt{D_{1}+m_{1} m_{1}^{*}} E^{-1}\right) \\
& +\operatorname{Tr}\left(E^{-*} F^{*}\left(D_{2}+m_{2} m_{2}^{*}\right) F E^{-1}\right) \\
& +\operatorname{Tr}\left(D_{2}+m_{2} m_{2}^{*}\right)
\end{aligned}
$$

Let $W=\sqrt{D_{2}+m_{2} m_{2}^{*}} F E^{-1} E^{-*} \sqrt{D_{1}+m_{1} m_{1}^{*}}$. Then, it is straightforward to see that $\widehat{L}$ and $\widehat{K}$ such that

$$
\widehat{L} \widehat{K}^{*}=W^{*}{\sqrt{\left(W W^{*}\right)}}^{-1}
$$

minimize the right side of the previous expression. Then solving (5) is equivalent to solving

$$
\min _{m_{1}, m_{2}} g\left(m_{1}, m_{2}\right)
$$

where

$$
\begin{array}{r}
g\left(m_{1}, m_{2}\right)=\operatorname{Tr}\left(E^{-*}\left(D_{1}+m_{1} m_{1}^{*}\right) E^{-1}\right)-2 \sqrt{W W^{*}} \\
+\operatorname{Tr}\left(E^{-*} F^{*}\left(D_{2}+m_{2} m_{2}^{*}\right) F E^{-1}\right)+\operatorname{Tr}\left(D_{2}+m_{2} m_{2}^{*}\right) \\
W=\sqrt{D_{2}+m_{2} m_{2}^{*}} F E^{-1} E^{-*} \sqrt{D_{1}+m_{1} m_{1}^{*}} .
\end{array}
$$

Let $\hat{m}_{1}, \hat{m}_{2}$ be the optimal solutions of (14) and let $\widehat{\Delta_{1}}, \widehat{\Delta_{2}}$ be the optimal solutions of (3). In the following section we show how from $\hat{m}_{1}, \hat{m}_{2}$ and the optimality conditions derived in [18] $\widehat{\Delta_{1}}$ and $\widehat{\Delta_{2}}$ can be found.

\section{OPTIMALITY CONDITIONS}

Let $\widehat{Z}$ be the optimal solution of (5). Then the optimality conditions from [18] read as

$$
\begin{gathered}
\left(\Theta_{1}^{*} \widehat{Z} \Theta_{1}-D_{1}\right) \widehat{\Delta_{1}}=\hat{m_{1}} \hat{m}_{1}{ }^{*} \widehat{\Delta_{1}}=0 \\
\left(\Theta_{2}^{*} \widehat{Z} \Theta_{2}-D_{2}\right) \widehat{\Delta_{2}}=\hat{m_{2}} \hat{m}_{2}{ }^{*} \widehat{\Delta_{2}}=0 \\
\widehat{Z}-\widehat{Z} \Theta_{1} \widehat{\Delta_{1}} \Theta_{1}^{*}-\widehat{Z} \Theta_{2} \widehat{\Delta_{2}} \Theta_{2}^{*}=0 .
\end{gathered}
$$

Before solving (17) let us compute its terms $\widehat{Z}, \Sigma_{1}=$ $\widehat{Z} \Theta_{1} \widehat{\Delta_{1}} \Theta_{1}^{*}$, and $\Sigma_{2}=\widehat{Z} \Theta_{2} \widehat{\Delta_{2}} \Theta_{2}^{*}$.

$$
\begin{aligned}
\Sigma_{1}= & \left(\Theta_{2} \sqrt{D_{2}+\hat{m}_{2} \hat{m}_{2}{ }^{*}} \widehat{K}+\Theta_{2}^{\perp} \widehat{S}\right) \\
& \left(\widehat{K}^{*} \sqrt{D_{2}+\hat{m}_{2} \hat{m}_{2}^{*}} \Theta_{2}^{*}+\widehat{S}^{*} \Theta_{2}^{\perp *}\right) \\
& \left(\Theta_{2} F+\Theta_{2}^{\perp} E\right) \widehat{\Delta_{1}}\left(F^{*} \Theta_{2}^{*}+E^{*} \Theta_{2}^{\perp *}\right)
\end{aligned}
$$

After some computations we get

$$
\begin{aligned}
& \quad \Sigma_{1}= \\
& =\Theta_{2}\left(\left(D_{2}+\hat{m}_{2} \hat{m}_{2}{ }^{*}\right) F \widehat{\Delta_{1}} F^{*}+\sqrt{D_{2}+\hat{m}_{2} \hat{m}_{2}}{ }^{*} \widehat{K} \widehat{S^{*}} E \widehat{\Delta_{1}} F^{*}\right) \Theta_{2}^{*} \\
& \quad+\Theta_{2}^{\perp}\left(\widehat{S} \widehat{K}^{*} \sqrt{D_{2}+\hat{m}_{2} \hat{m}_{2}{ }^{*}} F \widehat{\Delta_{1}} F^{*}+\widehat{S S^{*}} E \widehat{\Delta_{1}} F^{*}\right) \Theta_{2}^{*} \\
& +\Theta_{2}\left(\left(D_{2}+\hat{m}_{2} \hat{m}_{2}{ }^{*}\right) F \widehat{\Delta}_{1} E^{*}+\sqrt{D_{2}+\hat{m}_{2} \hat{m}_{2}}{ }^{*} \widehat{K} \widehat{S}^{*} E \widehat{\Delta_{1}} E^{*}\right) \Theta_{2}^{\perp *} \\
& +\Theta_{2}^{\perp}\left(\widehat{S} \widehat{K}^{*} \sqrt{D_{2}+\hat{m}_{2} \hat{m}_{2}{ }^{*}} F \widehat{\Delta_{1}} E^{*}+\widehat{S} \widehat{S}^{*} E \widehat{\Delta_{1}} E^{*}\right) \Theta_{2}^{\perp *}
\end{aligned}
$$


Similarly we have

$$
\begin{aligned}
& \Sigma_{2}=\left(\Theta_{2} \sqrt{D_{2}+\hat{m}_{2} \hat{m}_{2}}{ }^{*}\right. \\
&\left(\widehat{K}^{*} \sqrt{D_{2}+\hat{m}_{2} \hat{m}_{2}^{*}} \Theta_{2}^{*}+\Theta_{2}^{\perp} \widehat{S}\right) \\
&\left.\widehat{S}^{*} \Theta_{2}^{\perp *}\right) \Theta_{2} \widehat{\Delta_{1}} \Theta_{2}^{*}
\end{aligned}
$$

and after some computations

$\Sigma_{2}=\Theta_{2}\left(D_{2}+\hat{m}_{2} \hat{m}_{2}{ }^{*}\right) \widehat{\Delta_{2}} \Theta_{2}^{*}+\Theta_{2}^{\perp} \widehat{S} \widehat{K}^{*} \sqrt{D_{2}+\hat{m}_{2} \hat{m}_{2}{ }^{*}} \widehat{\Delta}_{2} \Theta_{2}^{*}$

Of course we have also

$$
\begin{aligned}
\widehat{Z}=\left(\Theta_{2} \sqrt{D_{2}+\hat{m}_{2} \hat{m}_{2}^{*}} \widehat{K}+\Theta_{2}^{\perp} \widehat{S}\right) & \left(\widehat{K}^{*} \sqrt{D_{2}+\hat{m}_{2} \hat{m}_{2}{ }^{*}} \Theta_{2}^{*}+\widehat{S}^{*} \Theta_{2}^{\perp *}\right)
\end{aligned}
$$

and after some computations

$$
\begin{aligned}
& \widehat{Z}=\Theta_{2}\left(D_{2}+\hat{m}_{2} \hat{m}_{2}{ }^{*}\right) \Theta_{2}^{*}+\Theta_{2}^{\perp} \widehat{S} \widehat{S}^{*} \Theta_{2}^{\perp *} \\
& +\Theta_{2} \sqrt{D_{2}+\hat{m}_{2} \hat{m}_{2}{ }^{*}} \widehat{K} \widehat{S}^{*} \Theta_{2}^{\perp *}+\Theta_{2}^{\perp} \widehat{S} \widehat{K}^{*} \sqrt{D_{2}+\hat{m}_{2} \hat{m}_{2}{ }^{*}} \Theta_{2}^{*}
\end{aligned}
$$

Since $\widehat{Z}=\Sigma_{1}+\Sigma_{2}$ then equating the vector coefficients next to $\Theta_{2} \Theta_{2}^{\perp *}$ in $\widehat{Z}$ and $\Sigma_{1}+\Sigma_{2}$ we have

$$
\begin{aligned}
& \sqrt{D_{2}+\hat{m}_{2} \hat{m}_{2}{ }^{*}} \widehat{K}\left(\widehat{K}^{*} \sqrt{D_{2}+\hat{m}_{2} \hat{m}_{2}{ }^{*}} F+\widehat{S}^{*} E\right) \widehat{\Delta}_{1} E^{*}= \\
& \sqrt{D_{2}+\hat{m}_{2} \hat{m}_{2}^{*}} \widehat{K} \widehat{S}^{*}
\end{aligned}
$$

Combining (10) and the previous equation we finally obtain

$$
\begin{aligned}
& \widehat{\Delta_{1}}=E^{-1} E^{-*}- \\
& \sqrt{D_{1}+\hat{m}_{1} \hat{m}_{1}{ }^{*}}-1 \widehat{L} \widehat{K}^{*} \sqrt{D_{2}+\hat{m}_{2} \hat{m}_{2}{ }^{*}} F E^{-1} E^{-*}
\end{aligned}
$$

where $\widehat{L} \widehat{K}^{*}$ is a function of $\hat{m}_{1}, \hat{m}_{2}$ and is given in (13). In a similar manner equating the vector coefficients next to $\Theta_{2} \Theta_{2}^{*}$ we have

$$
\begin{aligned}
& \quad \widehat{\Delta_{2}}= \\
& I-\sqrt{D_{2}+\hat{m}_{2} \hat{m}_{2}{ }^{*}}-1 \hat{K}\left(\widehat{K}^{*} \sqrt{D_{2}+\hat{m}_{2} \hat{m}_{2}{ }^{*}} F+\widehat{S}^{*} E\right) \widehat{\Delta_{1}} F^{*} \\
& =I-\sqrt{D_{2}+\hat{m}_{2} \hat{m}_{2}{ }^{*}}-1 \widehat{K} \widehat{L}^{*} \sqrt{D_{1}+\hat{m}_{1} \hat{m}_{1}{ }^{*}} \widehat{\Delta_{1}} F^{*}
\end{aligned}
$$

where $\widehat{K} \widehat{L}^{*}=\left(\widehat{L} \widehat{K}^{*}\right)^{*}$ and $\widehat{L} \widehat{K}^{*}$ is given in (13). Clearly, given $\hat{m}_{1}, \hat{m}_{2}, \widehat{\Delta_{1}}$ and $\widehat{\Delta_{2}}$ can be obtained from equations (18) and (19). In the following section we determine $\hat{m}_{1}, \hat{m}_{2}$, $\widehat{\Delta_{1}}$, and $\widehat{\Delta_{2}}$.

\section{SOLVING THE PRIMAL AND DUAL PROBLEMS}

It is clear from $(3)$ that $\widehat{\Delta_{1}}$ and $\widehat{\Delta_{2}}$ can have different rank. It is not difficult to see that there are 6 different cases for the ranks of $\widehat{\Delta_{1}}$ and $\widehat{\Delta_{2}}$. In this section we analyze all of them and provide an explicit characterization of the optimal solutions.

\section{A. Rank-2 $\Delta$ 's}

If $\widehat{\Delta_{1}}$ and $\widehat{\Delta_{2}}$ both have rank 2 then from (15) and (16) it easily follows that $\hat{m}_{1}=\hat{m}_{2}=0$. Then $\widehat{\Delta_{1}}$ and $\widehat{\Delta_{2}}$ can easily be obtained from (18) and (19).

\section{B. One of $\Delta$ 's is zero}

These two cases are straightforward. Directly from (3) it follows that if $\widehat{\Delta_{2}}=0$ then $\widehat{\Delta_{1}}=I$. Also if $\widehat{\Delta_{1}}=0$ it easily follows $\widehat{\Delta_{2}}=I$.

\section{One of $\Delta$ 's has rank 2, the other rank 1}

Without loss of generality we will assume that $\widehat{\Delta_{1}}$ is of rank one and $\widehat{\Delta_{2}}$ is of rank two. The case when $\widehat{\Delta_{2}}$ is of rank one and $\widehat{\Delta_{1}}$ is of rank two is completely symmetric.

If $\widehat{\Delta_{1}}$ is of rank one then from (15) we have that $\hat{m}_{1}$ is $2 \times 1$ vector. Furthermore, from (16) we have that $\hat{m}_{2}=0$. Then (14) can be simplified to

$$
\min _{m_{1}} g\left(m_{1}\right)
$$

where

$$
\begin{array}{r}
g\left(m_{1}\right)=\operatorname{Tr}\left(E^{-*}\left(D_{1}+m_{1} m_{1}^{*}\right) E^{-1}\right) \\
-2 \sqrt{\sqrt{D_{2}} F E^{-1} E^{-*}\left(D_{1}+m_{1} m_{1}^{*}\right) E^{-1} E^{-*} F^{*} \sqrt{D_{2}}} \\
+\operatorname{Tr}\left(E^{-*} F^{*}\left(D_{2}\right) F E^{-1}\right)+\operatorname{Tr}\left(D_{2}\right) .
\end{array}
$$

Furthermore, solving (20) is equivalent to solving

$$
\min _{v} \operatorname{Tr}\left(v B v^{*}\right)-2 \operatorname{Tr} \sqrt{D+v v^{*}}
$$

where $S=\sqrt{D_{2}} F E^{-1}, S E^{-*} D_{1} E^{-1} S^{*}=U D U^{*} v=$ $U^{*} S E^{-*} m_{1}, B=U^{*} S^{-*} S^{-1} U, U$ is a unitary matrix and $D$ is diagonal matrix. Clearly if $\hat{v}$ is a solution of (21) then $\hat{m}_{1}=E^{*} S^{-1} U \hat{v}$. Without loss of generality we can assume $v=\left[\begin{array}{c}\sqrt{v_{1}} \\ \sqrt{v_{2}} e^{j \phi}\end{array}\right], v_{1}, v_{2}$ are real, and $v_{1} \geq 0, v_{2} \geq 0$. Further, let $B=\left[\begin{array}{cc}b_{11} & b_{12} e^{j \beta} \\ b_{12} e^{-j \beta} & b_{22}\end{array}\right], D=\left[\begin{array}{cc}d_{1} & 0 \\ 0 & d_{2}\end{array}\right]$, and $b_{12} \geq 0$. After some algebraic transformations (21) can be written as

$$
\begin{aligned}
& \min _{v_{1}, v_{2}, \phi} v_{1} b_{11}+v_{2} b_{22}+2 \sqrt{v_{1} v_{2}} b_{12} \cos (\phi+\beta) \\
& -2 \sqrt{d_{1}+d_{2}+v_{1}+v_{2}+2 \sqrt{v_{1} d_{2}+v_{2} d_{1}+d_{1} d_{2}}} .
\end{aligned}
$$

Let $\hat{v_{1}}, \hat{v_{2}}, \hat{\phi}$ be the optimal solution of (22). Then clearly, $\hat{\phi}=-\beta+\pi$ and $(22)$ becomes

$$
\begin{aligned}
& \min _{v_{1}, v_{2}} v_{1} b_{11}+v_{2} b_{22}-2 \sqrt{v_{1} v_{2}} b_{12} \\
& -2 \sqrt{d_{1}+d_{2}+v_{1}+v_{2}+2 \sqrt{v_{1} d_{2}+v_{2} d_{1}+d_{1} d_{2}}} .
\end{aligned}
$$

Quite remarkably it can be shown that the previous problem is convex. Hence the optimal solution can be found after derivation. Let $x=d_{1}+d_{2}+v_{1}+v_{2}, y=v_{1} d_{2}+v_{2} d_{1}+d_{1} d_{2}$, and $\mathcal{L}=v_{1} b_{11}+v_{2} b_{22}-2 \sqrt{v_{1} v_{2}} b_{12}-2 \sqrt{x+2 \sqrt{y}}$. Then we have

$$
\begin{aligned}
& \frac{\partial \mathcal{L}}{\partial v_{1}}=b_{11}-\sqrt{v_{2} / v_{1}} b_{11}-\frac{1+\frac{d_{2}}{\sqrt{y}}}{\sqrt{x+2 \sqrt{y}}}=0 \\
& \frac{\partial \mathcal{L}}{\partial v_{2}}=b_{22}-\sqrt{v_{1} / v_{2}} b_{11}-\frac{1+\frac{d_{1}}{\sqrt{y}}}{\sqrt{x+2 \sqrt{y}}}=0 .
\end{aligned}
$$

Let $k=\sqrt{\frac{v_{2}}{v_{1}}}$. After some algebraic transformations from (24) we obtain

$$
\begin{aligned}
h(k) & =\sqrt{y}=\frac{d_{2}\left(b_{22}-b_{12} / k\right)-d_{1}\left(b_{11}-k b_{12}\right)}{b_{11}-k b_{12}-\left(b_{22}-b_{12} / k\right)} \\
v_{1} & =\left(h(k)^{2}-d_{1} d_{2}\right) /\left(d_{2}+k^{2} d_{1}\right) .
\end{aligned}
$$


Replacing (25) in (24) we finally have

$$
b_{11}-k b_{12}-\frac{1+\frac{d_{2}}{h(k)}}{d_{1}+d_{2}+\left(1+k^{2}\right) \frac{h(k)^{2}-d_{1} d_{2}}{d_{2}+k^{2} d_{1}}+2 h(k)}=0 .
$$

Let $\min _{k}=\min \left\{-b_{22}+\sqrt{\left(b_{11}-b_{22}\right)^{2}+4 b_{12}^{2}},-b_{22} \frac{d_{2}}{d_{1}}+\right.$ $\left.\sqrt{\left(b_{11}-b_{22} \frac{d_{2}}{d_{1}}\right)^{2}+4 b_{12}^{2} \frac{d_{2}}{d_{1}}}\right\}$ and $\max _{k}=\max \left\{-b_{22}+\right.$ $\left.\sqrt{\left(b_{11}-b_{22}\right)^{2}+4 b_{12}^{2}},-b_{22} \frac{d_{2}}{d_{1}}+\sqrt{\left(b_{11}-b_{22} \frac{d_{2}}{d_{1}}\right)^{2}+4 b_{12}^{2} \frac{d_{2}}{d_{1}}}\right\}$. Since $k \geq 0$ and $h(k) \geq 0$, it can be shown that optimal $\hat{k}$ is the unique solution of (26) from the interval $\left[\frac{b_{11}+\min _{k}}{2 b_{12}}, \frac{b_{11}+\max _{k}}{2 b_{12}}\right]$. This solution can then easily be obtained (e.g. using bisection method). Then $\hat{v}_{1}$ can be obtained from (25). Finally, we have $\hat{v}_{2}=\hat{k}^{2} \hat{v}_{1}, \hat{m}_{1}=$ $E^{*} S^{-1} U\left[\begin{array}{c}\sqrt{\hat{v_{1}}} \\ \sqrt{\hat{v}_{2}} e^{j(-\beta+\pi)}\end{array}\right]$, and $\widehat{\Delta_{1}}, \widehat{\Delta_{2}}$ from (18), (19). This concludes the case when one of $\Delta$ 's is of rank 1 and the other one is of rank 2 .

\section{Both $\Delta$ 's are rank 1}

When $\widehat{\Delta_{1}}$ and $\widehat{\Delta_{2}}$ are rank one we solve directly the primal problem given in (3). Let $\Delta_{1}=\delta_{1} \delta_{1}^{*}$ and $\Delta_{2}=\delta_{2} \delta_{2}^{*}$. Then (3) becomes

$$
\begin{aligned}
\max _{\delta_{1}, \delta_{2}} & \operatorname{Tr}\left(\delta_{1}^{*} D_{1} \delta_{1}\right)+\operatorname{Tr}\left(\delta_{2}^{*} D_{2} \delta_{2}\right) \\
\text { subject to } & \Theta_{1} \delta_{1} \delta_{1}^{*} \Theta_{1}^{*}+\Theta_{2} \delta_{2} \delta_{2}^{*} \Theta_{2}^{*} \leq I .
\end{aligned}
$$

It is not difficult to show that for optimal $\delta_{1}$ and $\delta_{2}$ holds $\delta_{2}^{*} \Theta_{2}^{*} \Theta_{1} \delta_{1}=0$ and $\delta_{1}^{*} \delta_{1}=\delta_{2}^{*} \delta_{2}=1$. Let $\Theta_{2}^{*} \Theta_{1}=P \Sigma Q^{*}$ where $P P^{*}=Q Q^{*}=I$ and $\Sigma=\left[\begin{array}{cc}1 / \sigma_{1} & 0 \\ 0 & 1 / \sigma_{2}\end{array}\right]$. Let $s_{1}=Q^{*} \delta_{1}, s_{2}=P^{*} \delta_{2}$, and let $s_{2}^{\perp}$ be unit norm vector such that $s_{2}^{*} s_{2}^{\perp}=0$. Further, let $s_{2}=\left[\begin{array}{c}a \\ \sqrt{1-a^{2}} e^{j \psi}\end{array}\right], 0 \leq$ $a \leq 1$. Then it easily follows that $s_{1}=\Sigma^{-1} s_{2}^{\perp} \xi$ where $\xi=1 / \sqrt{s_{2}^{\perp *} \Sigma^{-*} \Sigma^{-1} s_{2}^{\perp}}=1 / \sqrt{\sigma_{1}^{2}-a^{2}\left(\sigma_{1}^{2}-\sigma_{2}^{2}\right)}$. Let $M=\Sigma^{-*} Q^{*} D_{1} Q \Sigma^{-1}=\left[\begin{array}{cc}m_{11} & m_{12} e^{j \gamma_{2}} \\ m_{12} e^{-j \gamma_{2}} & m_{22}\end{array}\right], P^{*} D_{2} P=$ $\left[\begin{array}{cc}d_{11} & d_{12} e^{j \gamma_{1}} \\ d_{12} e^{-j \gamma_{1}} & d_{22}\end{array}\right], m_{12} \geq 0$, and $d_{12} \geq 0$. After some algebraic transformations (27) can be written as

$$
\begin{gathered}
\max _{0 \leq a \leq 1, \psi} \frac{m_{11}\left(1-a^{2}\right)+m_{22} a^{2}}{\sigma_{1}^{2}-a^{2}\left(\sigma_{1}^{2}-\sigma_{2}^{2}\right)}+a^{2} d_{11}+\left(1-a^{2}\right) d_{22} \\
+2 d_{12} \sqrt{a^{2}-a^{4}} \cos \left(\psi+\gamma_{1}\right)-\frac{2 \sqrt{a^{2}-a^{4}} m_{12} \cos \left(\psi+\gamma_{2}\right)}{\sigma_{1}^{2}-a^{2}\left(\sigma_{1}^{2}-\sigma_{2}^{2}\right)} .
\end{gathered}
$$

The optimal $\psi$ can be given as $\cos \hat{\psi}=c_{1} / \sqrt{c_{1}^{2}+c_{2}^{2}}, c_{1}=$ $2 d_{12} \cos \gamma_{2}-\frac{2 m_{12} \cos \gamma_{1}}{\sigma_{1}^{2}-a^{2}\left(\sigma_{1}^{2}-\sigma_{2}^{2}\right)}, c_{2}=\frac{2 m_{12} \sin \gamma_{1}}{\sigma_{1}^{2}-a^{2}\left(\sigma_{1}^{2}-\sigma_{2}^{2}\right)}-2 d_{12} \sin \gamma_{2}$. Then (28) simplifies to $\max _{0 \leq a \leq 1} \mathcal{F}(a)$

where

$$
\begin{gathered}
\mathcal{F}(a)=\frac{m_{11}\left(1-a^{2}\right)+m_{22} a^{2}}{\sigma_{1}^{2}-a^{2}\left(\sigma_{1}^{2}-\sigma_{2}^{2}\right)}+a^{2} d_{11}+\left(1-a^{2}\right) d_{22} \\
+\sqrt{a^{2}-a^{4}} \sqrt{\left(\frac{2 m_{12}}{\sigma_{1}^{2}-a^{2}\left(\sigma_{1}^{2}-\sigma_{2}^{2}\right)}-2 d_{12} \cos \gamma\right)^{2}+4 d_{12}^{2} \sin ^{2} \gamma} .
\end{gathered}
$$

and $\gamma=\gamma_{1}-\gamma_{2}$. Further, let $z=\xi^{2}=1 /\left(\sigma_{1}^{2}-a^{2}\left(\sigma_{1}^{2}-\sigma_{2}^{2}\right)\right)$. Then (29) can be transformed to

$$
\frac{1}{\max \left\{\sigma_{1}^{2}, \sigma_{2}^{2}\right\}} \leq z \leq \frac{1}{\min \left\{\sigma_{1}^{2}, \sigma_{2}^{2}\right\}} \mathcal{F}(z)
$$

where

$$
\begin{aligned}
& \mathcal{F}(z)=\left(m_{11}+m_{22}\right) z \\
& +\frac{\left(d_{11}-m_{11} z\right)\left(\sigma_{1}^{2}-1 / z\right)+\left(d_{22}-m_{22} z\right)\left(1 / z-\sigma_{2}^{2}\right)}{\sigma_{1}^{2}-\sigma_{2}^{2}} \\
& +\frac{\sqrt{\left(\sigma_{1}^{2}-\frac{1}{z}\right)\left(\frac{1}{z}-\sigma_{2}^{2}\right)}}{\left|\sigma_{1}^{2}-\sigma_{2}^{2}\right|} \sqrt{\left(2 m_{12} z-2 d_{12} \cos \gamma\right)^{2}+4 d_{12}^{2} \sin ^{2} \gamma} .
\end{aligned}
$$

To find a solution to (30) we differentiate (31).

$$
\begin{gathered}
\frac{\partial \mathcal{F}}{\partial z}=\frac{m_{22} \sigma_{1}^{2}-m_{11} \sigma_{2}^{2}}{\sigma_{1}^{2}-\sigma_{2}^{2}}+\frac{d_{11}-d_{22}}{z^{2}\left(\sigma_{1}^{2}-\sigma_{2}^{2}\right)}+\frac{1}{\left|\sigma_{1}^{2}-\sigma_{2}^{2}\right|} \times \\
\frac{m_{12}^{2}\left(\left(\sigma_{1}^{2}+\sigma_{2}^{2}\right)+\frac{2 d_{12}}{m_{12}} \cos \gamma\left(\sigma_{1}^{2} \sigma_{2}^{2}-\frac{1}{z^{2}}\right)\right)-d_{12}^{2} \frac{z\left(d_{1}^{2}+d_{2}^{2}\right)-2}{z^{3}}}{\sqrt{\left(\sigma_{1}^{2}-\frac{1}{z}\right)\left(\frac{1}{z}-\sigma_{2}^{2}\right)} \sqrt{\left(m_{12} z-d_{12} \cos \gamma\right)^{2}+d_{12}^{2} \sin ^{2} \gamma}}=0 .
\end{gathered}
$$

Let $\hat{z}$ be a solution of (32). Then we have $\hat{a}=\frac{\left(\sigma_{1}^{2}-1 / \hat{z}\right)}{\sigma_{1}^{2}-\sigma_{2}^{2}}$, $\hat{s_{2}}=\left[\frac{\hat{a}}{\sqrt{1-\hat{a}^{2}}} e^{j \hat{\psi}}\right], \hat{s_{2}}{ }^{\perp}$ is a unit vector such that ${\hat{s_{2}}}^{{ }^{*}}{\hat{s_{2}}}^{\perp}=$ $0, \hat{\delta_{1}}=Q \Sigma^{-1}{\hat{s_{2}}}^{\perp} \sqrt{\hat{z}}$, and $\hat{\delta_{2}}=P \hat{s_{2}}$. Since in general there may be several (at most 8) solutions $\hat{z}$ of (32), we choose the one which produces $\hat{\delta_{1}}$ and $\hat{\delta_{2}}$ that maximize (3). This concludes the case of rank $1 \Delta$ 's.

\section{SUMMARY}

In this section we summarize the results from the previous section.

Lemma 1: Let $\widehat{\Delta_{1}}$ and $\widehat{\Delta_{2}}$ be the solutions of (3). Further assume that they are both of rank 2 . Then we have

$$
\begin{aligned}
& \widehat{\Delta_{1}}=E^{-1} E^{-*}-{\sqrt{D_{1}}}^{-1} \widehat{L} \widehat{K}^{*} \sqrt{D_{2}} F E^{-1} E^{-*} \\
& \widehat{\Delta_{2}}=I-{\sqrt{D_{2}}}^{-1} \widehat{K} \widehat{L}^{*} \sqrt{D_{1}} \widehat{\Delta}_{1} F^{*}
\end{aligned}
$$

where

$$
\begin{aligned}
& \widehat{L} \widehat{K}^{*}= \\
& \sqrt{D_{1}} E^{-1} E^{-*} F^{*} \sqrt{D_{2}} \sqrt{\sqrt{D_{2}} F E^{-1} E^{-*} D_{1} E^{-1} E^{-*} F^{*}{\sqrt{D_{2}}}^{-1}} \text {. }
\end{aligned}
$$

Proof: Follows from the previous discussion.

Lemma 2: Let $\widehat{\Delta_{1}}$ and $\widehat{\Delta_{2}}$ be the solutions of (3). Further assume that $\widehat{\Delta_{1}}$ is of rank 2 and $\widehat{\Delta_{2}}=0$. Then we have

$$
\widehat{\Delta_{1}}=I, \widehat{\Delta_{2}}=0 \text {. }
$$

Similarly if $\widehat{\Delta_{2}}$ is of rank 2 and $\widehat{\Delta_{1}}=0$ we have

$$
\widehat{\Delta_{1}}=0, \widehat{\Delta_{2}}=I \text {. }
$$

Proof: Follows from the previous discussion.

Lemma 3: Let $\widehat{\Delta_{1}}$ and $\widehat{\Delta_{2}}$ be the solutions of (3). Further assume that $\widehat{\Delta_{1}}$ is of rank 1 and $\widehat{\Delta_{2}}$ is of rank 2 . Then $\widehat{\Delta_{1}}$ and $\widehat{\Delta_{2}}$ are given by (18) and (19) respectively, where $\hat{m_{1}}=E^{*} S^{-1} U\left[\begin{array}{c}\sqrt{\hat{v_{1}}} \\ \sqrt{\hat{v}_{2}} e^{j(-\beta+\pi)}\end{array}\right], \hat{v_{2}}=\hat{k}^{2} \hat{v_{1}}, \hat{k}$ is the unique solution of (26) from the interval $\left[\frac{b_{11}+m i n_{k}}{2 b_{12}}, \frac{b_{11}+m a x_{k}}{2 b_{12}}\right]$, $\hat{v}_{1}=\left(h(\hat{k})^{2}-d_{1} d_{2}\right) /\left(d_{2}+\hat{k}^{2} d_{1}\right), h(k)$ is as introduced 
in (25), $E, S, U, \beta, b_{11}, b_{12}$ are as introduced below (21), and $\min _{k}, \max _{k}$ are as introduced below (26).

Proof: Follows from the previous discussion.

If $\widehat{\Delta_{1}}$ is of rank 2 and $\widehat{\Delta_{2}}$ is of rank 1 then they can be determined in a similar fashion. However, since this case is completely symmetric to the one that we have already analyzed in the interest of saving the space we omit its analysis here.

Lemma 4: Let $\widehat{\Delta_{1}}$ and $\widehat{\Delta_{2}}$ be the solutions of (3). Further assume that they are both of rank 1 . Then $\widehat{\Delta_{1}}=\hat{\delta}_{1} \hat{\delta}_{1}{ }^{*}$ and

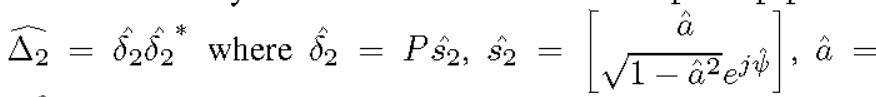
$\frac{\left(\sigma_{1}^{2}-1 / \hat{z}\right)}{\sigma_{1}^{2}-\sigma_{2}^{2}}, \hat{\delta_{1}}=Q \Sigma^{-1}{\hat{s_{2}}}^{\perp} \sqrt{\hat{z}},{\hat{s_{2}}}^{\perp}$ is a unit vector such that ${\hat{s_{2}}}^{*}{\hat{s_{2}}}^{\perp}=0, P, \Sigma, Q$ are as defined below (27), $\hat{\psi}$ is as defined below (28), and $\hat{z}$ is solution of (32) which produces $\hat{\delta_{1}}, \hat{\delta_{2}}$ that maximize (3).

Proof: Follows from the previous discussion.

We unify the previous lemmas in the following theorem.

Theorem 1: Let $\widehat{\Delta_{1}}$ and $\widehat{\Delta_{2}}$ be the solutions of (3). Then they correspond to those $\widehat{\Delta_{1}}$ and $\widehat{\Delta_{2}}$ from the previous lemmas which maximize (3).

Proof: First we note that the ranks of $\widehat{\Delta_{1}}$ and $\widehat{\Delta_{2}}$ can be at most 2. It is also easy to see that the cases when sum of their ranks is less than 2 can never happen. Hence there are only 6 cases left and they are all covered by previous lemmas. Which of these 6 cases is solution is determined according to the value of the objective in (3) that they produce. The one which produces the largest value of objective in (3) is the solution. This ends the proof.

\section{UNAMBIGUOUS DISCRIMINATION BETWEEN PURE} AND MIXED STATE

In this section we briefly look at the unambiguous discrimination between a pure and a mixed state. This problem was also considered earlier in [4]. Here we provide solution based on the framework developed earlier in the paper. The problem formulation is again as in (3), i.e.

$$
\max _{\Delta_{1}=\Delta_{1}^{*} \geq 0, \Delta_{2}=\Delta_{2}^{*} \geq 0} \sum_{i=1}^{2} p_{i} \operatorname{Tr}\left(\rho_{i} \Theta_{i} \Delta_{i} \Theta_{i}^{*}\right) .
$$

However, the dimensions of the matrices $\Theta_{1}, \Theta_{2}, \Delta_{1}, \Delta_{2}$ are now different. Namely, $\Theta_{2}$ is an $(l+1) \times 1$ unit norm vector, $\Theta_{1}$ is a $(l+1) \times l$ matrix such that $\Theta_{1}^{*} \Theta_{1}=I, \Delta_{1}$ is $l \times l$ hermitian positive semi-definite matrix, $\Delta_{2}$ is a positive scalar. As earlier $D_{i}=\Theta_{i}^{*} p_{i} \rho_{i} \Theta_{i}, i=1,2$, and $F=\Theta_{2}^{*} \Theta_{1}$. (Note that now $D_{2}$ is a scalar and $\mathrm{F}$ is a row vector.) Mimicking the procedure given earlier in the paper the following theorem can be proved.

Theorem 2: Let $\widehat{\Delta_{1}}$ and $\widehat{\Delta_{2}}$ denote the solution of (33). Then it holds

$$
\begin{aligned}
& \widehat{\Delta_{1}}=I-\left(\frac{\sqrt{D_{2}+\hat{m}_{2} \hat{m}_{2}{ }^{*}}}{\sqrt{F\left(D_{1}+\hat{m}_{1} \hat{m}_{1}{ }^{*}\right) F^{*}}}-1\right) \frac{F^{*} F}{1-F F^{*}} \\
& \widehat{\Delta_{2}}=1-\frac{\sqrt{F\left(D_{1}+\hat{m}_{1} \hat{m}_{1}{ }^{*}\right) F^{*}}}{\sqrt{D_{2}+\hat{m}_{2} \hat{m}_{2}{ }^{*}}\left(1-F F^{*}\right)}+\frac{F F^{*}}{1-F F^{*}}
\end{aligned}
$$

where $\hat{m}_{1}$ and $\hat{m}_{2}$ are depending on the values $D_{1}, D_{2}$, and $F$ given as

$$
\begin{cases}\hat{m}_{1}=0, \hat{m}_{2}=\sqrt{F D_{1} F^{*}-D_{2}}, & D_{2} \leq F D_{1} F^{*} \\ \hat{m}_{1}=\frac{\sqrt{D_{2}\left(F F^{*}\right)^{2}-F D_{1} F^{*} F^{*}}}{F F^{*}}, \hat{m}_{2}=0, & D_{2} \geq \frac{F D_{1} F^{*}}{\left(F F^{*}\right)^{2}} \\ \hat{m}_{1}=0, \hat{m}_{2}=0, & \text { otherwise }\end{cases}
$$$$
\text { Proof: Omitted. }
$$

It is not difficult to check that the solution given in Theorem 2 matches the one obtained in [4] in the context of quantum filtering.

\section{CONCLUSION}

We considered the problem of distinguishing unambiguously between two general mixed quantum states of rank 2. We provided an explicit analytical characterization of the optimal measurement operators. Additionally, using the developed framework we derived an analytical solution for the unambiguous discrimination of a pure and a mixed quantum state.

\section{ACKNOWLEDGMENT}

This work was supported in part by the National Science Foundation under grant no. CCR-0133818, by the David and Lucille Packard Foundation, and by Caltech's Lee Center for Advanced Networking.

\section{REFERENCES}

[1] I.D.Ivanovic, "How to differentiate between non-orthogonal states," Phys. Lett. A, vol. 123, 1987.

[2] D. Dieks, "Overlap and distinguishability of quantum states," Phys. Lett. A, vol. $126,1988$.

[3] A. Peres, "How to differentiate between non-orthogonal states," Phys. Lett. A, vol. 128, 1988.

[4] J. Bergou, U. Herzog, and M. Hillery, "Quantum state filtering and discrimination between sets of Boolean functions," Physical Review Letters 90, 2579012003.

[5] J. Bergou, U. Herzog, and M. Hillery, Lect. Notes Phys. 649, 417-465 (Springer, Berlin, 2004).

[6] J. Bergou, E. Feldman, and M. Hillery, "Optimal unambiguous discrimination of two subspaces as a case in mixed state discrimination," Phys. Rev. A, vol. 73, 0321072006 .

[7] T.Rudolph and R.W.Spekkens and P.S.Tumer, "Unambiguous discrimination of mixed states," Phys. Rev. A,vol 682003.

[8] Y.C.Eldar, "A Semidefinite Programming Approach to Optimal Unambiguous Discrimination of Quantum States," IEEE Trans. Inform Theory,vol. 49, Feb. 2003.

[9] Y.C.Eldar, "Mixed quantum state detection with inconclusive results," Phys. Rev. A, vol. 67, Apr. 2003

[10] J. Fiurasek and M.Jezek, "Optimal discrimination of mixed quantum states involving inconclusive results," Phys. Rev. A, 2003.

[11] P. Raynal and N. Lutkenhaus, "Reduction theorems for optimal unambiguous state discrimination of density matrices," Phys. Rev. A, Aug. 2003.

[12] P. Raynal and N. Lutkenhaus, "Optimal unambiguous state discrimination of two density matrices: Lower bound and class of exact solutions," Phys. Rev. A 72, 2005

[13] U. Herzog, "Optimal unambiguous discrimination of two mixed states and application to states connected by a class of unitary transformations," http://arxiv.org/abs/quant-ph/0611087, Nov. 2006.

[14] G. Wang and M. Yang, "Unambiguous discrimination among quantum operations," Physical Rev. A, Apr. 2006

[15] C. Zhang, Y. Feng, and M. Ying, "Unambiguous discrimination of mixed quantum states," Physics Letters A, May 2006.

[16] Y. Feng, R. Duan, and M. Ying, "Unambiguous discrimination between quantum mixed states," Physical Rev. A, 2004.

[17] X. F. Zhou, Y. S. Zhang, G. C. Guo, "Unambiguous discrimination between two mixed states," http://arxiv.org/abs/quant-ph/0611095, Nov. 2006.

[18] Y. Eldar, M. Stojnic, and B.Hassibi, "Optimum quantum detectors for unambiguous detection of mixed states," Physical Rev. A, Jun 2004 